\section{Chancengleichheit in der} Forschungsförderung

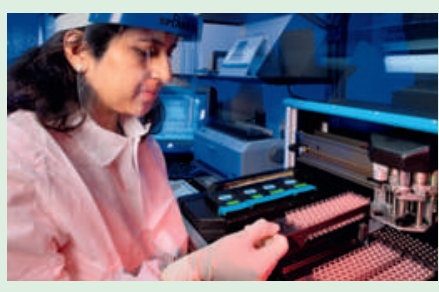

Frauen sind in der Wissenschaft und Forschung untervertreten.

Der Forschungsrat des SNF lädt Forschende, Fachpersonen und Interessierte zur Konferenz «Gender and Excellence - Challenges in Research Funding» ein. Ziel ist es, neue Wege zur Verwirklichung der Chancengleichheit in der Forschungsförderung zu finden. Frauen sind in TopPositionen der Wissenschaft und Forschung noch immer klar untervertreten. Infolgedessen sind auch die Förderungsgelder ungleich auf die Geschlechter verteilt. Was läuft falsch im System und wo liegen die Schwierigkeiten? Diese Fragen werden an der öffentlichen Konferenz am 22. Oktober in Bern diskutiert.

(SNF)

\section{La cigarette électronique}

Le concours Expérience non-fumeur va bientôt commencer pour l'année scolaire 2014-2015. Les cigarettes électroniques sont un thème d'actualité. Elles semblent tout à fait inoffensives aux élèves, et pourtant nombre d'adolescents vivent à travers elles un premier rituel qui peut les inciter à continuer. Ensuite, il n'y a qu'un petit pas à faire pour passer au tabagisme traditionnel. Le concours est ouvert à toutes les classes de Suisse, de la $6^{e}$ à la $9^{e}$ année. Les classes qui y participent s'engagent à ne consommer aucun produit du tabac et - c'est la nouveauté de cette édition 2014-2015 aucune cigarette électronique.

(Association suisse pour la prévention du tabagisme)

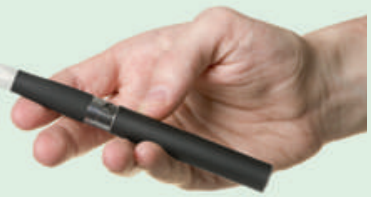

Les cigarettes électroniques sont un thème d'actualité.

\section{Neuntausend Franken für aidskranke Menschen in Simbabwe}

Zum zwölften Mal hat im September «Der Ärzteball» stattgefunden. Die Gäste, Sponsoren und das Ballkomitee spendeten zusammen neuntausend Franken zu Gunsten von Swiss Aids Care International (SACI). Der Schweizer Aids-Pionier Prof. Ruedi Lüthy hat in Zimbabwe unter schwierigen Bedingungen eine ambulante Klinik und ein Ausbildungszentrum aufgebaut. Heute werden in der Newlands Clinic rund 5000 Patientinnen und Patienten umfassend betreut. In Simbabwe waren bis anhin - wie in vielen anderen Drittweltländern - nur HIV-Medikamente der ersten und zweiten Generation erhältlich. Nun sind neu auch Medikamente der dritten Generation verfügbar. Die Newlands Clinic gehört zu den vier Kliniken, welche diese Therapie künftig anbieten dürfen.

(Ärzteball/SACI)

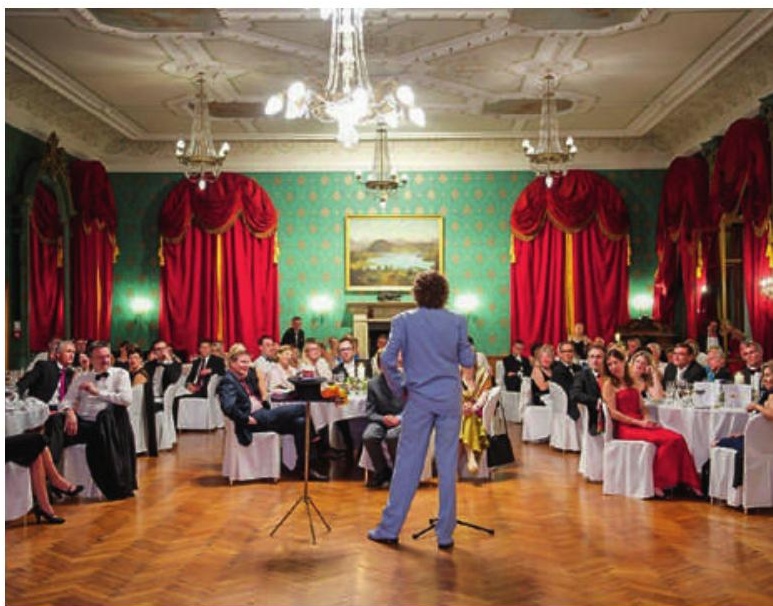

Magisch: Toni Rey verblüffte die Gäste am Ärzteball mit seinen Künsten.

\section{Jeux de hasard}

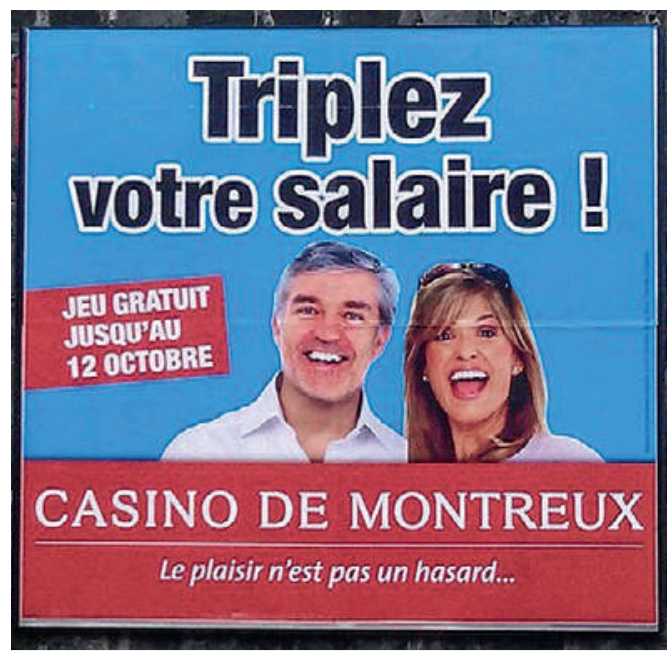

Difficile de résister à cette tentation..

A coup d'affiches grand format, le casino de Montreux s'est efforcé d'attirer de nouveaux joueurs et joueuses. A travers le slogan «Triplez votre salaire!», il a ciblé clairement des personnes qui ne gagnent pas assez et qui ont peut-être du mal à joindre les deux bouts. Des personnes qui rêvent de toucher le jackpot pour garder la tête hors de l'eau. Difficile de résister à la tentation de jouer quand on vous fait de surcroît miroiter une partie gratuite. Or, ce sont justement ces personnes qui sont les plus exposées au risque de développer un comportement problématique à l'égard du jeu. Une nouvelle loi sur les jeux d'argent a été mise en chantier par le Conseil fédéral. Addiction suisse a demandé au Conseil fédéral et au Parlement d'introduire, entre autres, l'interdiction des jeux gratuits et de la publicité personnalisée dans la nouvelle loi.

(Addiction suisse)

\section{Million Kinder sterben an ihrem ersten Lebenstag}

Die Zahl der Kinder, die vor ihrem fünften Geburtstag sterben, konnte um die Hälfte gesenkt werden. Dennoch sterben jährlich immer noch fast 2,8 Millionen Neugeborene in den ersten vier Wochen ihres Lebens, eine Million davon an ihrem ersten Lebenstag. Dies sind Ergebnisse aus dem neuen UNICEF-Bericht zur weltweiten Kindersterblichkeit. Viele dieser Todesfälle könnten mit einfachen und kostengünstigen Massnahmen vor, während und unmittelbar nach der Geburt verhindert werden. Der Bericht zeigt zudem auf, dass neben dem Alter auch das Bildungsniveau und der Wohlstand einen grossen Einfluss auf die Überlebenschancen von Neugeborenen haben. Die Säuglingssterblichkeitsrate ist bei Müttern ohne Ausbildung fast doppelt so hoch wie bei
Müttern, die eine weiterführende Schule besucht haben.

(Unicef)

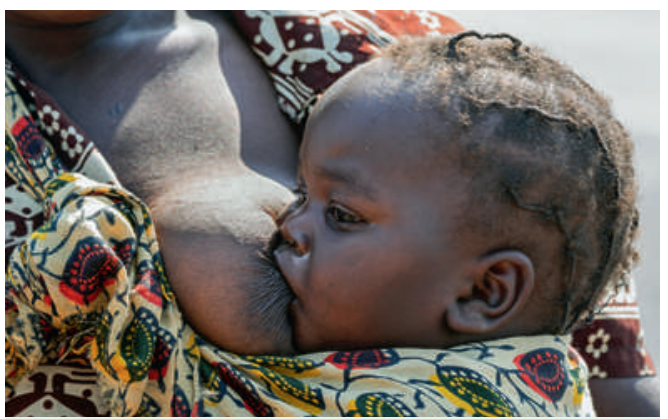

Afrika gehört zu den Regionen der Erde mit hoher Kindersterblichkeit. 